\title{
Non-destructive Analysis of Food Adulteration and Legitimacy by FTIR Technology
}

Khan U*, Afzaal M, Arshad MS and Imran M

Department of Food Sciences, Government College University Faisalabad, Pakistan

\begin{abstract}
Despite of old methods, an emerging technology of Fourier Transform Infrared (FTIR) spectroscopy has been appeared to use as a promising application for the detection of adulteration in food. FTIR method works on the principal of interaction of electromagnetic radiations with the molecules of specific food with defined energy. In this review, the three regions of electromagnetic spectra including near infrared, mid infrared and far infrared are the points of spotlight. For food industry, FTIR method is expedient, automatic, time saving and most importantly, it shows non-destructive analysis of food physically as well as chemically. Moreover, FTIR in collaboration with the authentic commendable data analytical system appeared as a tremendous appeal for descriptive test of food from extremely large quantity to its trivial component level, showing dramatically potential for adulteration analysis with the substantial contrivance for the quality control progressions. A systematic review of literaturehas been performed to amass the information of FTIR technique as non destructive method for analysis of intentionally added harmful chemicals from existing research publications.
\end{abstract}

Keywords: FTIR; Food adulteration; NIR; MIR; FIR; Food safety; Food authenticity

\section{Introduction}

Food Adulteration is performed to achieve more quantity of the food in order to make more profit, following the addition of such chemical substances which should not be present legally or having unwanted status by the recipient. It is astonishing that impostors are at stride ahead of food safety organizations; their escalating technologies protect and allow them to become more refine in their work with each passing moment $[1,2]$. As on one side food is adulterated to get additional profit while on the other hand it imposes detrimental aspects to the human health and has become a global threat which is to be eradicated as soon as possible, for that we have to first detect it. There are various methods for adulteration analysis but they have to follow the destruction of specific lot or specific area of foodstuff, in modern era with growing economic as well as quality standards we need to develop or discover such technique which do not impart any qualitative change into the product while analyzing. As for the sake of food safety we have to analyze its adulteration with the intention of keeping its quality as it is, hence our scientists discovered an attractive substitute analytical technique for the non-destructive analysis of food adulteration named as "Fourier transform infrared (FTIR) technique".

At one side if we protect food from exterior damage by providing antimicrobial packaging [3], it leads us towards the safe side of prepared food but there is still a question mark that, is the inside packaged food is safe? Or is adulterated with some harmful substances ultimately causing health hazards? Hence, there is foremost need to develop or to study upon the techniques which indicate the adulteration and approve the legitimacy of packaged food. It has gained attention as it is easy to operate, slight sample preparation required, perilous solvent utilization is reduced and is time and cost effective process. The present study compiles facts and figures on the application of FTIR in food technology as numerous researches have been performed for this submission. The basic understanding of mechanism regarding spectroscopy smooths the progress of recognizing the application of infrared practice for detection of food adulteration. In this method, infrared spectra is generated by observing various absorptions by the molecules absorbing infrared radiations as a form of energy which cause their mechanical motion in vibration as well as in rotation form [4,5]. FTIR spectroscopy has vast area for analysis as it analyzes even from very small molecular complex level to a large tissue level with the mapping of cellular components including carbohydrates, fats and proteins [6,7]. While analyzing a certain assay, definite functional groups, which could be amide or carbonyl group, present in the molecule shows typical infrared absorption at their particular short range of frequency despite of their association with other molecules. Position of band showed by the infrared absorption spectrum, occasionally influenced by neighboring atoms of vibrational group, changes with the change in the energy of vibrational motion due to the complex interaction among atoms of molecule, each of which vibrates in its own transition. Accordingly, an infrared spectrum is employed to identify samples with the knowledge of measuring functional groups as well $[4,5]$. Infrared spectroscopy examines the outcome of interaction among radiated energy and matter in the infrared range, and the effect is appraised by quantifying the absorption of different infrared frequencies through a sample positioned in the infrared beam pathway. The radiation can either be transmitted or absorbed, relying up on the frequency of it and the molecular structure in the sample, when a beam consisting of infrared radiation is passed from the sample. Infrared radiation enlivens certain molecules, hence creating vibrations from the thrilled status at rigid wavelengths. When the energy quanta are provided, the molecular mechanical behavior is changed, hence changing their rotational as well as vibrational modes. The energy absorption exhibits at different frequencies parallel to the molecular means of vibration of the analogous chemical group or molecule [8].

*Corresonding author: Khan U, Department of Food Sciences, Government College University Faisalabad, Pakistan, Tel: 92419201266; E-mail uroojkhan162@gmail.com

Received October 12, 2015; Accepted November 02, 2015; Published November 06, 2015

Citation: Khan U, Afzaal M, Arshad MS, Imran M (2015) Non-destructive Analysis of Food Adulteration and Legitimacy by FTIR Technology. J Food Ind Microbiol 1: 103. doi: $10.4172 / 2572-4134.1000103$

Copyright: (c) $2015 \mathrm{Khan} U$, et al. This is an open-access article distributed under the terms of the Creative Commons Attribution License, which permits unrestricted use, distribution, and reproduction in any medium, provided the original author and source are credited. 
The infrared electromagnetic spectrum ranges from 14000 to 50 $\mathrm{cm}^{-1}$, and being categorized in three sub categories:

\section{a) Near infrared \\ b) Mid infrared \\ c) Far infrared}

The near infrared region lies in the range of 14000 to $4000 \mathrm{~cm}^{-1}$, while mid infrared occupies the region of 4000 to $400 \mathrm{~cm}^{-1}$, and far infrared ranges from 400 to $50 \mathrm{~cm}^{-1}$. In the present study we mainly focus on first two regions as these gain prominent role in the detection of adulterants by understanding the trend of electromagnetic radiations and their interaction with the molecules [9].

\section{Near Infrared (NIR) Spectra}

Near infrared spectra formed as consequence of comparatively weak and wide overtones and the fundamental combination bands of vibrational motions related chiefly with carbon-hydrogen $(\mathrm{C}-\mathrm{H})$, Nitrogen-hydrogen $(\mathrm{N}-\mathrm{H})$ and oxygen-hydrogen $(\mathrm{O}-\mathrm{H})$ functional groups. In other words, near infrared bands are considered to be the outcome of chemical bonds which are likely to be diverging from harmonicity and show intricate vibrational transits. Near infrared absorption strength reduces with the escalating overtone grade, resulting by the band which arises from the transitions over two or three following first or second overtone respectively, or advanced energy intensity ( 12500 to $4000 \mathrm{~cm}^{-1}, 800$ to $2500 \mathrm{~nm}$ ) of fundamental vibrational frequency. Moreover, near infrared spectra arise when two or more vibrations combine at the same time as a result of particular photon absorption $[10,11]$.

Near infrared spectra show least structural discrimination. Unfortunately when various combination bands and overtones show superposition in NIR region then it causes extremely low selectivity of structure as compared to the spectra of MIR. Hence, for the NIR measurement in contrast to MIR, by increasing the total thickness of sample we can recompense with the low intensity of NIR absorption. The measurement of NIR follows millimeters to centimeter unit while that of MIR follow micrometer, thus MIR fundamental bands are 11 about 10 to 100 times more intense as compared to their corresponding NIR bands. This can be used for the samples that are strongly light scattering or highly absorbing with no dilution or widespread sample preparation [12]. The fourier transform NIR instruments made their way to the food industry by having potent ability for the process control and chemical quality assessment as they have good austerity with good flexibility for handling either they could analyze the samples in glass covering as well. Moreover, the food having more moisture is more readily analyzed [10].

\section{Mid Infrared (MIR) spectra}

In the case of organic compounds MIR spectra is usually employed for structural fingerprinting (recognition) due to the structural bands resulting from the primary vibrations related to particular functional group [5]. The bands in the fingerprinting section of polysaccharides, proteins, lipids and carotenoids are located in the region of MIR ranges 1,200 to $700 \mathrm{~cm}^{-1}$ with high resultant information of structure. The MIR region spectra can also be used for the quantitative analysis as the bands intensities increase or decrease with the respective functional group concentration with direct proportion [13].

\section{The uplift of FTIR over conventional IR techniques}

The accuracy and speed of the action has been amplified when some monochromators and typical conservative prism were replaced by fourier transform instrumentation, it boost up the infrared technology with qualities. For the measurement of manifold frequencies at a time FTIR employed interferometric modulation of the radiation which results in intefrogram which is again analyzed by using complex algorithms for resultant original spectra. The problem of over lapping bands of IR usually transpire during the analysis of some complex samples can be solved by escalating the peak height with the dropping the bandwidth [14]. Additionally FTIR involves less equipment mechanical wear. The resolution in FTIR instrumentation is not determined through the beam size, it can be calculated by the travel or stroke of moveable mirror and its resultant data within a short time [4]

\section{Attenuated Total Reflectance (ATR)}

The combination of FTIR with attenuated total reflectance (ATR) allowed the collection of spectra from semisolids, solids, thin films and liquids. In comparison to conventional IR practice, ATR is provided with a fast tool allowing minimum sample preparation, gives more accurate and quality data within no time with material identification as well [15]. It is basically a reflection technique in which infrared light, when applied to the sample, is internally reflected by internal reflecting element surface giving high index refraction. A resultant standing wave, called evanescent wave, is created when radiation pass through the crystals, hence the sample which contain crystals also contact with evanescent wave by absorbing the radiation and give the respective resultant spectra for the detection and the wave (absorbs the radiation) is attenuated by the absorbance of sample, hence is called as Attenuated total reflectance (ATR) [16].

\section{FTIR Appliance in the Legitimacy and Detection of Food Adulterants}

It has been an extremely imperative point for regulatory agencies, retailers, food processors and specially for consumers that the certification/verification/authentication of produce should be well defined by its variety, commodity or geographical origin as pricey ingredients are more prone to fraudulent or adulteration or unintentional mislabeling. So, the demand of this era is a novel rapid technique which legalizes these statements. FTIR has appeared as a promising tool in this critical situation and is applicable in herbal produce, fruit juices, dairy, agricultural products, edible oils and others, and being applied as FT-Near-Infrared and FT-Mid-Infrared with various statistical methods. FTIR has a vast array of benefits including less time requirement (hardly 1 to 2 minutes), with a single spectrum great number of adulterants can be detected, with this high speed it has no requirement of sample preparation as well, hence produce minimum waste material. Additionally, it doesn't destroy the sample which is recognized as its best benefit from regulatory point of view [17].

\section{Application of FT-NIR Spectroscopy}

Adulteration in herbal products: The Immuno stimulant herbal supplements are made up with the help of Echinacea species E. purpurea(L.) Moench, E. pallid and E. angustifolia, while their commercial preparations are adulterated by the roots of various Echinacea species or Parthenium integrifolium L. which ultimately impart negative effect on the efficiency of Echinacea products strongly [18]. FT-NIR spectroscopy has been accounted for the snappy identification of E. purpurea roots and the detection of echinacoside substance [19]. By utilizing FT-NIR spectroscopy the vicinity of other Echinacea species can be identified at least 10\% defilement [20]. 
Inferable from the low amount of echinacosides in the most precious E. purpurea roots in contrast to E. pallida and E. angustifolia [19], partial least-square (PLS) calculations utilizing NIR spectra produce powerful models for the fast and reliable screening of E. purpurea in the preparation of herbal products.

Adulteration in natural oils: Dietary supplement oils (DSOs, for example, grape-seed oil, evening primrose oil, burageseed oil, and flax oil) are adulterated with less expensive and less advantageous oils, and this disastrous situation has turned into food safety as well as food quality issue. Misclassifications of bonafide oils can be come about because of the varieties present in distinctive brands of the same oil due to the assortment, processing conditions and plant origin including the oil sorts having fundamentally the similar composition or same creations. It has been reported that DSOs are being adulterated with cheaper food oils by $2 \%$ to $20 \% \mathrm{v} / \mathrm{v}$ [21].

Adulteration in juices: An outstanding use of FT-NIR spectroscopy has been reported for the detection of juices, syrups and purees for adulteration. These items are every now and again contaminated with less expensive juice concentrates, beet sugars, corn, cane, and syrups for extra financial profit. Scientists documented the utilization of FT-NIR and the factorial discriminant analysis to determine the adulteration of orange juice with grapefruit juice, orange pulp-wash, and artificial sugar/acid blend [22].

Exact grouping rates $>90 \%$ were resolved for adulterated orange juice at $50 \mathrm{~g} / \mathrm{kg}$ or more, with no tainted orange juice being anticipated as reliable. Contal et al. demonstrated that adulteration of raspberry or strawberry juice with the juice of apple could be noticed at levels $>10 \%$ by employing partial least square near infrared (PLS-NIR) models [23]. The amount of sugar in non-disseminating juices can be determined by transmittance NIR spectra precisely and accurately [24], though by FT-NIR transflectance data, the forecast mistakes for scrambling juice samples could be improved [25]. Furthermore, the obstruction from the wide and powerful vibrational bands of water in NIR calculations of watery frameworks can be reduced by rapid solvent removal and estimation of the dry concentrate by employing diffuse reflectance spectroscopy $[26,27]$. Application of FTIR in the determination of adulteration level in apple juice is also promoted by Duraisamy et al. in his latest study of 2015 [28].

\section{Application of FT-MIR Spectroscopy}

FT-MIR spectroscopy has been utilized for the authentication of juice of high-esteem components tainted with that of inferior sources. Pomegranates have been lauded for their antioxidant potential and against prostate cancer they are known for their potential chemopreventative impact. FT-MIR spectra have been utilized to separate unadulterated pomegranate juice content from juice provided with grape juice content $(2 \%-14 \% \mathrm{v} / \mathrm{v})$, here employing the principle component analysis (PCA) and 1,780-1,685 $\mathrm{cm}^{-1}$ ( $\mathrm{C}=\mathrm{O}$ extending) IR region [29]. Moreover, FT-MIR has also been effective in the separation of varieties of different fruits and their geological inceptions. A vital parameter for observing legitimacy in fruit preps, jams and purees is the fruit content percentage, while least requirements for every sort of item have been ascertained. A partial least-squares regression (PLSR) relating the fruit and the FTIR spectra fixated on a band at $1729 \mathrm{~cm}^{-1}$ providing with excellent calibration statistics $(\mathrm{R} 2=0.94)$ while used for strawberry jam [30,31]. He et al. studied apple juices, concord grapes, cranberries, plum nectar blend and blueberries from a variety of producers. Figure 1 recapitulates the applications of MIR spectroscopy for food legitimacy using PLSR model provided with their results.

\section{Regression}

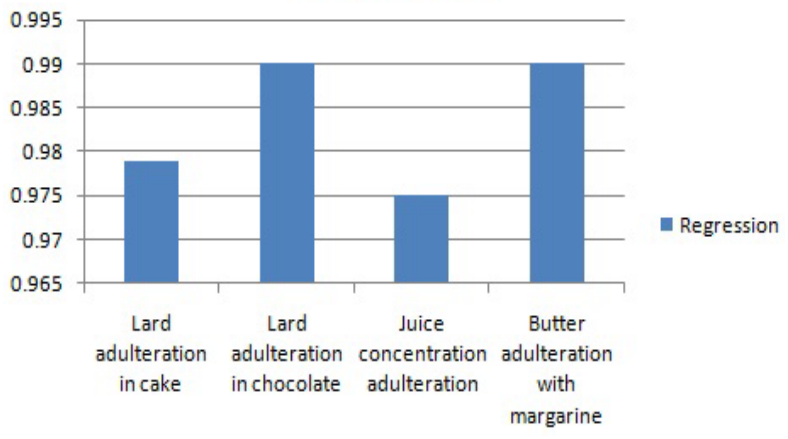

Figure 1: MIR application for food legitimacy using PLSR model and its results.

Spectral data obtained from the solid phase extraction of fruit juices showed the improved pattern identification [following the algorithm of soft independent analysis of class analogy (SIMCA)] as compared to the data obtained from pure juice, which is subjected to the differentiation of juice content on the base or fruit origin. The impedance of sugar on the spectra is diminished by solid phase and separated out the phenolic content which shows unique fingerprint to juice legitimacy. There are some restrictions of this technique, including the extraction strategy and the requirement for a more extensive scope of tests to enhance the strength of the model, yet noticed that this system was a change over going before endeavors [32]. Here the question arises that why we don't use old or conventional methods for the verification of juices, the answer lies in the fact that some old or conventional methods like, carbon isotope ratio analysis or chromatography employed for the validation of juices are now not pose to be practicable due to the employment of harmful solvents, and also is time consuming strategy, not viable for quality control settings and just one parameter can be checked at a time.

Adulteration in honey: Honey has been termed as a characteristic substance delivered from honeybees and the adulteration of sugars negates this description, and its dilution with complex or simple sugars is commonly observed as adulteration in common market [33]. This is quite hard to identify this corruption because adulterants copy the common sugar profile of honey, which comprises of $31.2 \%$ of glucose and $38.2 \%$ of fructose. Honey adulteration is likewise hard to recognize due to the vast variability in the item because of the environment and flower, honeybee species, development and storage or processing conditions. Nowadays, honey transparency is analyzed via carbon isotope ratio test, which is time consuming and expensive. Thus, to check the authenticity of honey FT-MIR technique is used, but the spectra should be redressed against water background to make accurate water signals overlapping the signals of solute. The analysis was centered at the region that communicates the sugars (800-1500 $\mathrm{cm}^{-1}$ ). Usually honey is adulterated with $7 \%$ to $25 \%$ invert sugar, glucose, sucrose and fructose. Classification of complex and simple sugars was $100 \%$ accomplished by PLS and LDA data compression respectively. Consolidating the honey of multiple assortments obliged more factors while lowering the achievement rate to $95.5 \%$. More work must be accomplished to incorporate the honey of various origins, with numerous adulterants [34]. Another study took a gander at artisanal adulteration of honey with diverse variety of sugar syrups including high fructose corn syrup (HFCS), dextrose syrup, invert beet syrup, partial invert cane syrup and beet sucrose. 
The classification of about $95 \%$ of pure honey, dextrose and beet sucrose samples was carried out by some models however these models were not proved to be proficient in the detection of adulterants like HFCS or invert syrup [35]. Thus, FT-MIR was utilized to assess the natural origin of honey by correlating the pollen investigation. Similarly as with numerous sustenance items, honey produced in specific areas is valued for its glorious sensory qualities and is sold at a high cost. To successfully recognize the root of the item, MIR could be employed as a screening tool; however it would be necessary for it to be coalesced with extra testing techniques. Moreover, MIR did not prove to be helpful in quantitative identification of hydroxylmethyl furan (HMF) as a pointer of heat injury to the produce. FTIR with the combination of ATIR is also applicable in the authentication and determination of adulteration in honey [36,37].

Adulteration in milk and milk products: Conventional techniques for checking milk legitimacy depend up on wet science to focus the measure of a artificial compound in a suspected stuff and a resulting examination of the values acquired by the identical material [38]. There is a broad use of both NIR and MIR in cheese legitimacy with ATRIR spectroscopy, these procedures can possibly help the processors to stick to progressively strict food legitimacy legislation. By using IR spectroscopy, the classification of Emmental cheeses from distinctive origins was accounted by Picque et al. [39]. The discrimination of the various sorts of Emmental cheeses with the accuracy of $78 \%$ is empowered by the data obtained from ATR-IR spectroscopy, while $87 \%$ precision was acquired by employing the transmission spectra of concentrates. The IR has potential to differentiate as indicated by the source of milk utilized for processing. In another study, Pillonel et al. reported the consequences of an extensive screening analysis into the legitimacy of Emmental cheese and its origin detection. 100\% classification is delivered by NIR diffuse reflectance by gathering into the six districts of cheese source, though MIR transmittance got $100 \%$ accurate discrimination when contrasting Switzerland and alternate areas pooled as an assemblage [40].

The identity standard of butter obliges that the vegetable oil must not be present in it and in this manner, the insertion of margarine abuses this claim. Bright obvious (UV/Vis) spectroscopy, Gas chromatography (GC), mass spectrometry (MS) and NMR are present strategies for the validation of butter and these techniques have the identification limits of $2 \%$ to $5 \%$, however they are time intensive and expensive. IR spectroscopy consolidated with PLSR-created models that assessed mixing of butter with levels of margarine starting from $0 \%$ to $100 \% \mathrm{v} / \mathrm{v}$ with SECV less than $1.2 \%$ and $\mathrm{R} 2>0.99$. With the lesser intensity of adulteration ranges ( $0 \%$ to $5 \%, 0 \%$ to $25 \%$, and $20 \%$ to $60 \%$ ), the advancement of chemometric systems has enhanced the power of the models [40].

Adulteration in wines: Patrons nowadays are reflecting their careful consideration on natural or organic items and have eager to pay a premium cost for these articles. This propensity is recognized by wine industry, thus vineyards around the globe are now creating numerous organic wines. In the starting of 2009 , there was no steady wine-industry system that would encourage organic wine adulteration and legitimacy to be easily and ably determined. Therefore, Cozzolino et al. was the first to take a gander at utilization of a strategy for the classification of organic and inorganic commercial wines. Utilizing MIR consolidated with direct discriminant examination (LDA), PCA and discriminant PLS (DPLS) regression, almost 200 specimens of white and red wines from 13 locales in Australia were examined. LDA accurately classified $75 \%$ of organic wines, while DPLS classified $85 \%$. While the PCA score plot classified both the organic and inorganic wines, yet there was a small overlap [41]. A fine differentiation among the samples delivered under organic and inorganic production strategies was permitted by MIR consolidated with chemometric systems. Investigation of the PLS loadings did not demonstrate any specific individual chemical consideration that clarified the classification between wines, relatively numerous chemical compounds, such as volatile, nonvolatile and phenolic compounds could contribute to the classification of organic to inorganic wines.

Additionally, maple syrup and nectar items are focuses for corrupt producers to make benefit by the addition of less expensive beet and cane sugars. In maple syrup, FT-NIR spectroscopy in collaboration with quantitative (PLSR and principle component regression) and discriminant [(LDA and canonical variate analysis (CVA)] analysis, for the classification purpose of adulterants has been effectively employed [42]. Models created with FTIR calculations were well suitable for the quantitative analysis of the vicinity of adulterants as compared to dispersive NIR measurements. Relative results have been accounted for the analysis of oregano, thyme and chamomile crucial oils by dispersive NIR and ATR-IR spectroscopy [20].

Adulteration in fats and oils: FTIR spectroscopy has potent prospective to analyze the adulteration of lard. Syahariza et al. assessed lard adulterated shortening with the levels of $0 \%$ to $100 \%$ working in collaboration with PLSR models in the regions of $1117 \mathrm{~cm}^{-1}$ to $1097 \mathrm{~cm}^{-1}$ and $990 \mathrm{~cm}^{-1}$ to $950 \mathrm{~cm}^{-1}$, delivering a model with $\mathrm{R} 2=0.9790$ along with a standard calibration error of 1.75 [43]. Additionally, ATRIR consolidated with PLSR had the capacity to identify cocoa butter adulterated with lard from $0 \%$ to $15 \%$ with $\mathrm{R} 2=0.99$ along with 1.305 as a standard error [44]. Olive oil adulteration was dated in the times of Roman Empire, showing such an aged history of adulteration [45]. In the mid-1990s, rise of spectroscopy for olive oil validation was concerned and a generous measure of work has been devoted to utilize the strategy for the detection of adulteration of extra virgin olive oil. The band of $3,009 \mathrm{~cm}^{-1}$ has been observed for the measurement of adulterants, that the stature of this band for extra virgin olive oil is minor than that of different sorts of oils and changes as per the degree of the adulteration. With a nearly $6 \%$ as the detection limit a high relationship coefficient (over 0.99) was built up. Preceding PCA Wavelet compression delivers $5 \%$ as detection limit for mixtures of binary oils [46]. By employing the first measure of the FTIR spectra in the fingerprinting area and PLSR $(\mathrm{SECV}=0.285$ and $\mathrm{R} 2=0.999)$ extra virgin olive oil having palm oil as an adulterant can be perceived [47]. Various degrees of unsaturation, chain length differences in acyl moieties and different sorts of triglyceride substitution patterns were reflected by FTIR spectra [5]. To affirm the origin assert, extra virgin olive oil has been the focus of studies. The European Union provides extra virgin olive oil makers with the labels reflecting ensured description of geographical origin. For the classification of samples with the accuracy of up to $90 \%$ the nuclear attractive reverberation (NMR) has been employed, however this strategy is time consuming and expensive. $80 \%$ of the classification is achieved by mean-focused first and second imitative spectra joined with PLSR on the premise of origin which pose to be perfect for screening on account of the high throughput of the technique [48].

\section{FTIR Appliance to Detect Potentially Harmful Adulterants}

Food adulteration is carried out by the addition of cheap and low quality material while its smaller part comprises of intentional addition 
of hazardous materials. Either the adulteration of food products is occurred involuntarily during any production practice, such as acryl amide and trans fat, or by unintentional addition of injurious material in the product such as melamine. A substantial approach has been publicized by the means of progress in multivariate data analysis systems and FTIR spectroscopy to determine the modification in the food composition which can thus be analyzed to reveal the addition of harmful substances in the stuff.

Health affairs associated with Trans fat has been nominated recently for the world. The primary source of trans fat in commercial food products is partial hydrogenated oil, however a very small amount of trans fat is recognized in dairy and meat based products naturally [49]. For low saturated fat containing oils, the hydrogenation practice is highly beneficial because this process authorizes this oil to be employed in the place of saturated fats, however trans fats are formed by the partial hydrogenation process. The increasing threat for heart diseases has been aroused by the means of trans fats that which are well-known for lowering the high density lipoprotein (HDL) cholesterol and increasing low density lipoprotein (LDL) cholesterol. In the beginning of 2006, it was required, at nutritional charts, to illustrate the amount of trans fats which are present in the supplements of food, moreover gram per serving of them should be described as well. Lesser than $0.5 \mathrm{~g}$ are termed as trans fats. It is producer's responsibility to make certain the legitimacy of certified nutritional information of the item that is in food and drug administration's (FDA's) technique. The primary method for analysis through GC incorporates the extraction of fats, volatile fatty acid methyl ester (FAME) derivation employment, identification of mixture which is capable of categorizing the FAME components with a column, entreating every single trans FAME, accumulating the reaction of detector and lastly, changing the tri-glycerol alternates [50]. These methodologies require a high degree prepared experts because each part needs to be quantitative or delegated.

Since 1950's IR strategy has been utilized to look at the amount of separated trans double bonds in fats and oils and this system is relied on IR bonds at $966 \mathrm{~cm}^{-1}$ resulting to $\mathrm{CH}$ out bonds in fats and oils, in light of the fact that this band is particular for trans double bond confinement. The face with the utilization of this system contains the identification of trans band from those due to because of impedance included to other functional groups or conjugated double bonds. Because of convergence of groups, fats and oils that have trans fats in low sum are for the most part influenced. On the raised slanting pattern trans fat are set up and by the lessening in the level of trans fats the tallness estimations and exactness of region is likewise diminished.

To flatten the inclining benchmark, two official strategies of ATRFTIR (AOAC strategy 2000.10 and AOCS Cd 14d-99) consolidate a foundation of trans fats. By utilizing trans free reference oil as a part of which triladians are included, conference standards are developed. To create an alignment bend on $65^{\circ} \mathrm{C}$ single or multibounce ATR cell, the guidelines are investigated. Obscure trans amount that is spoken to as percent of aggregates fat, can be standardized by the utilizing a curve. There may be opposite influence on the exactness of the model by the utilization of reference oil disagree essentially from the mix of obscure specimen, fundamentally underneath $5 \%$ of aggregate fat [49]. There is another unconstructive second subordinate ATR-FTIR procedure that claims to upgrade the exactness and precision of quantitative level of trans fats in nourishment test that is at time approved by global study. The tallness of unconstructive second subordinate with respect to demeanor of trans assimilation is evaluated through this system. From transmonoenetrielaidin mixed with tripalmitin, reference principles are created and to find out the aggregate fat substance in eatable oils and fats this fast procedure is immaculate.

Concerning the wellbeing of the items, quality issue was perceived by a dairy organization. An approach was created for the location and quantization of a remote material found in the milk intake filter by utilizing a system which is similar to a great degree with that of melamine identification. The IR range of the remote material was formed and composed with business piece rodent bait TOM CATR, giving similar IR patterns of absorption. Once the outside material had been perceived, the worry moved to the quality of the milk. The dairy organization has keen interest in the assessment of milk for conceivable adulteration with the outside material. Micro spectroscopy (IRMS) is the technique which permit the analysis of sample with very small area ranges few micrones [50]. For biological analysis, it follows the combined effect of 2 analytical techniques, connecting infinity corrected microscope (ICM) with an excellent infrared spectrometer supplied with mercury-cadmium-telluride detector (MCTD) which ultimately produce less noise with the spectrum usually about 10 to 100 times than that of produced by deuterated triglycine sulfate detector (DTGSD).

The new era of potent MIR spectroscopic synthetic imaging systems joins step-scan FT Michelson interferometry with indium antimonide focal/central plane range (FPA) picture identification. The IR focal plane image indicator gives an instrumental multiplex/ multichannel favorable position, empowering spectra at all pixels to be gathered at the same time, while the interferometer segment of the framework permits all the otherworldly frequencies to be measured simultaneously. This top quality strategy speaks to the eventual fate of infrared chemical imaging examination, which consolidates the capacity of spectroscopy for atomic investigation with the potential of representation or visualization. IR imaging permits the exact portrayal of the composition of chemical, structure of domain, and compound construction modeling of an assortment of substances, data frequently pivotal for the consideration of complex samples [4].

\section{Conclusion}

FTIR has been emerged as a promising technique for the detection of food adulterants and their legitimacy. This is less time consuming and an efficient method eradicating the past problems of industrial members. Nowadays, industry has facing serious problem in the detection of their raw samples, either they are in pure form for their final product or not, they are required to follow old and more time consuming crude methods which ultimately trends the industry in economical loss. This technique, described in present study ultimately boost $u$ the industrial era by making the detection of intentional or accidental contamination problems. The review illustrates about the ability of FTIR spectroscopy itself as well as its combined effects to achieve the resolution of food adulteration detection, it is a well established, high throughput, rapid and non-destructive analytical technique through which wide range of sample are examined provided with fingerprint character of chemical as well as biochemical substance present in given sample. With minimum background training FTIR spectroscopy can boost the food industry with quick and precise tackles for the analysis of food adulteration with the reliable appraisal of food safety and quality. It will surely facilitate the food manufacturer to quickly assess the authentication of their food, providing with the allowance of timely rectification in measures throughout manufacturing process.

\section{References}

1. Lakshmi V (2012) Food Adulteration. Guntur, Andhra Pradesh, India. 
Citation: Khan U, Afzaal M, Arshad MS, Imran M (2015) Non-destructive Analysis of Food Adulteration and Legitimacy by FTIR Technology. J Food Ind Microbiol 1: 103. doi:10.4172/2572-4134.1000103

2. Arianna R (2015) "Application of Fourier Transform Infrared (FTIR) Spectroscopy in the Characterization of Tannins," Applied Spectroscopy Reviews 50: 407-442.

3. Afzaal, Urooj Khan, Sakandar HA, Akhtar MN (2015) Antmicrobial Packaging, a Step towards Safe Food: A Review. Global Journal of Science Frontier Research 15: 2 .

4. Diem M (1993) Introduction to Modern Vibrational Spectroscopy. New York: Wiley.

5. Guillen M, Cabo N (1999) Infrared spectroscopy in the study of edible oils and fats. J Sci Food Agric. 75: 1-11.

6. Levin IW, Bhargava R (1997) Fourier transforms infrared vibrationa spectroscopic imaging: Integrating microscopy and molecular recognition. Ann Rev Phys Chem 56: 429-474.

7. Petibois C, De'le'ris G (2006) Chemical mapping of tumor progression by FTIR imaging: Towards molecular histopathology. Trends Biotechnol 24: 455-462.

8. Alvarez-Ordóñez A, Prieto M (2012) Technical and methodological aspects of fourier transform infrared spectroscopy in food microbiology research. In Fourier Transform Infrared Spectroscopy in Food Microbiology, 1-18. Springer US.

9. Rodriguez-Saona LE, Allendorf ME (2011) Annu. Rev. Use of FTIR for Rapid Authentication and Detection of Adulteration of Food. Food Sci Technol 2: 467-483.

10. Osborne BG (2000) Near-Infrared Spectroscopy in Food Analysis. Chichester UK: Wiley,

11. Barton FE II (2002) Theory and principles of near infrared spectroscopy. NIR Spectrosc 14: 2-18

12. Shenk JS, Workman JJ, Weterhans MO (2001) Application of NIRS to agricultural products. In Handbook of Near-Infrared Analysis (eds) Burns DA New York: Marcel Dekker, Ciurczak EW 16: 419-474.

13. Pare JRJ, Belanger JMR (1997) Instrumental Methods in Food Analysis. New York: Elsevier.

14. Markovich RJ (1991) Introduction to Fourier transform infrared spectroscopy and applications in the pharmaceutical sciences. Pharm. Res 8: 663-75.

15. Pike Technol (2010) ATR theory and applications.

16. PerkinElmer (2004) ATR accessories An overview.

17. Kelly JF, Downey G (2005) Detection of sugar adulterants in apple juice using Fourier-transform infrared spectroscopy and chemometrics. J Agric Food Chem 53: 3281-3286.

18. Laasonen M, Harmia-Pulkkinen T, Simard CL, Michiels E, RasanenM et al. (2002a) Fast identification of Echinacea purpurea dried roots using nearinfrared spectroscopy. Anal Chem 74: 2493-2499.

19. Laasonen M, Wennberg T, Harmia-Pulkkinen T, Vuorela H (2002b) Simultaneous analysis of alkamides and caffeic acid derivatives for the identification of Echinacea purpurea, Echinacea angustifolia, Echinacea pallid and Partheniumintegrifoliumroots. Planta Med 68: 572-574.

20. Schulz H, Schrader B, Quilitzsch R, Pfeffer S, Kruger H, et al. (2003) Rapid classification of basil chemotypes byvarious vibrational spectroscopy methods. J Agric Food Chem 51: 2475-2481.

21. Ozen B, Weiss I, Mauer JL (2003) Dietary supplement oil classification and detection of adulteration using Fourier transform infrared spectroscopy. J Agric Food Chem 51: 5871-5876.

22. Twomey M, Downey G, McNulty PB (1995) The potential of NIR spectroscopy for the detection of the adulteration of orange juice. J Sci Food Agric 67: 77-84.

23. Contal L, Leon V, Downey G (2002) Detection and quantification of apple adulteration in strawberry and raspberry purees using visible and near infrared spectroscopy. J Near Infrared Spectrosc 10: 289-299.

24. Rodriguez-Saona LE, Allendorf ME (2011) Use of FTIR for Rapid Authentication and Detection of Adulteration of Food. Food Sci Technol 2: 467-483.

25. Segtnan VH, Isaksson T (2000) Evaluating near infrared techniques for quantitative analysis of carbohydrates in fruit juice model systems. J Near Infrared Spectrosc 8: 109-116.

26. Fischer WB, Eysel HH, Nielsen OF, Bertie EJ (1994) Corrections to the baseline distortions in the $\mathrm{OH}$-stretch region of aqueous solutions. Appl Spectrosc 48: 107-112.
27. Alfaro G, Meurens M, Birth GS (1990) Liquid analysis by dry extract nearinfrared reflectance on fiberglass. Appl. Spectrosc 44: 979-986.

28. Li W, Goovaerts P, Meurens M (1996) Quantitative analysis of individual sugars and acids in orange juices by near-infrared spectroscopy of dry extract. Agric Food Chem 44: 2252-2259.

29. Senbagam D et. al. (2015) Optimization of Lactobacillus brevis NS01 Brevicin Production and Its Application in Apple Juice Biopreservation Using FoodGrade Clarifying Agent Silica as a Carrier. Food and Bioprocess Technology 8: $1750-1761$.

30. Vardin H, Tay A, Ozen B, Mauer L (2008) Authentication of pomegranate juice concentrate using FTIRspectroscopy and chemometrics, Food Chem 108: 742 748

31. Fugel R, Carle R, Schieber A (2005) Quality and authenticity control of fruit purees, fruit preparations and jams: A review. Trends Food Sci. Technol 16: 433-441.

32. He J, Rodriguez-Saona LE, Giusti MM (2007) Midinfrared spectroscopy for juice authentication- rapid differentiationof commercial juices. J Agric Food Chem 55: 4443-4452.

33. Kelly JD, Petisco C, Downey G (2006) Application of Fourier transform midinfrared spectroscopy to the discrimination between Irish artisanal honey and such honey adulterated with various sugar syrups. J Agric 54: 6166-6171.

34. Anjos O (2015) Application of FTIR-ATR spectroscopy to the quantification of sugar in honey. Food chemistry 169: 218-223.

35. Gok S (2015) Differentiation of Anatolian honey samples from different botanical origins by ATR-FTIR spectroscopy using multivariate analysis. Food chemistry 170: 234-240.

36. Karoui R, Baerdemaeker DJ (2007) A review of the analytical methods coupled with chemometric tools forthe determination of the quality and identity of dairy products. Food Chem 102: 621-640.

37. Picque D, Cattenoz T, Corrieu G (2002) Discrimination of Emmental cheeses by infrared spectroscopy Milchwiss. Milk Sci Int 57: 202-204.

38. Pillonel L, Badertscher R, Butikofer U, Casey M, Dalla Torre M, et al. (2002) Analytical methods for thedetermination of the geographic origin of Emmentale cheese. Main framework of the project; chemical, biochemical, microbiological, color and sensory analyses. Eur. Food Res Technol 215: 260-267.

39. Koca N, Kocaoglu-Vurma NA, Harper WJ, Rodriguez-Saona LE (2010) Application of temperaturecontrolledattenuated total reflectance-mid-infared (ATR-MIR) spectroscopy for rapid estimation ofbutter adulteration. Food Chem 121: $778-782$.

40. Cozzolino D, Holdstock M, Dambergs RG, Cynkar WU, Smith PA (2009) Mid infrared spectroscopy andmultivariate analysis: $A$ tool to discriminate between organic and nonorganic wines grown in Australia. Food Chem 116: 761-765.

41. Paradkar MM, Sivakesava S, Irudayaraj J (2002) Discrimination and classification of adulterants in maple syrup with the use of infrared spectroscopic techniques. J Sci Food Agric 83: 714-721.

42. Syahariza ZA, Che Man YB, Selamat J, Bakar J (2005) Detection of lard adulteration in cake formulation by Fourier transforminfared (FTIR) spectroscopy. Food Chem 92: 365-371.

43. Che Man YB, Syahariza ZA, Mirghani MES, Jinap S, Bakar J, et al. (2005) Analysis of potential lard adulteration inchocolate and chocolate products using Fourier transform infrared spectroscopy. Food Chem 90: 815-819.

44. Ulberth F, Buchgraber M (2000) Authenticity of fats and oils. Eur J Lipid Sci. Technol 102: 687-694.

45. Gurdeniz G, Ozen B (2009) Detection of adulteration of extra virgin olive oil by chemometric analysis ofmid-infrared spectral data. Food Chem 116: 519-525.

46. Rohman A, Che Man YB (2010) FTIR spectroscopy combined with chemometrics for analysis of lard in themixtures with body fats of lamb, cow, and chicken. Int. Food Res J 17: 519-526.

47. Hennessy S, Downey G, O'Donnell CP (2009) Confirmation of food origin claims by Fourier transforminfrared spectroscopy and chemometrics: Extra virgin olive oil from Liguria 57: 1735-1741.

48. Rodriguez-Saona LE, Allendorf ME (2011) Use of FTIR for rapid authentication and detection of adulteration of food. Annual review of food science and technology: 467-483. 
Citation: Khan U, Afzaal M, Arshad MS, Imran M (2015) Non-destructive Analysis of Food Adulteration and Legitimacy by FTIR Technology. J Food Ind Microbiol 1: 103. doi:10.4172/2572-4134.1000103

Page 7 of 7

49. Mossoba MM, Moss J, Kramer JK (2009) Trans-fat labeling and levels in U.S foods: Assessment of chromatographicand infrared spectroscopic techniques for regulatory compliance. J Am Oil Chem Soc 92: 1284-1300.
50. Baeten V, Dardenne P (2002) Spectroscopy: Developments in instrumentation and analysis. Grasas Aceites 53: 45-63. 